\title{
ADESÃO DO IDOSO AO TRATAMENTO DA HIPERTENSÃO ARTERIAL SISTÊMICA: REVISÃO INTEGRATIVA*
}

\author{
Marina Mendes Soares ${ }^{1}$, Leonardo Oliveira Leão e Silva² ${ }^{2}$ Carlos Alberto Dias³, Suely Maria Rodrigues ${ }^{4}$, \\ Carla Jorge Machado
}

\begin{abstract}
RESUMO: Objetivando identificar na literatura os fatores que interferem no processo de adesão ao tratamento da hipertensão, em especial entre os idosos, foi realizada uma revisão integrativa de literatura. Utilizando-se as bases de dados SciELO, LILACS e PubMed foram selecionados 36 artigos, publicados entre janeiro de 2006 e dezembro de 2010, delimitando-se, a partir destes, cinco grandes grupos de fatores que interferem na adesão ao tratamento: regime terapêutico; aspectos socioeconômicos e demográficos; relação com os serviços e profissionais de saúde; aspectos psicossociais e culturais; e apoio familiar e social. Com base na literatura, pessoas do sexo masculino e idosos com baixa renda constituem as populações mais vulneráveis, sendo múltiplos os fatores que dificultam a adesão ao tratamento da hipertensão arterial. A adoção de uma abordagem multiprofissional no tratamento dos portadores de hipertensão constitui-se na abordagem mais adequada para promover a adesão ao tratamento. PALAVRAS-CHAVE: Hipertensão; Terapêutica; Idoso; Adesão à medicação.

\section{COMPLIANCE WITH TREATMENT FOR SYSTEMIC ARTERIAL HYPERTENSION AMONG THE ELDERLY: AN INTEGRATIVE REVIEW}

ABSTRACT: An integrative literature review was carried out with the aim of identifying in the literature the factors which interfere with the process of complying with treatment for hypertension, especially among the elderly. Using the data-bases SciELO LILACS and Pubmed, 36 articles were selected, published between January 2006 and December 2010. From these, five major groups of factors which interfere with compliance with treatment were defined: therapeutic regime: socio-economic and demographic aspects: relationship with the healthcare service and healthcare professionals: psycho-social and cultural aspects: and family and social support. According to the literature, elderly men on a low income are the most vulnerable population, there being multiple factors which impede compliance with treatment for arterial hypertension. The most appropriate step for promoting compliance with treatment is the adoption of a multiprofessional approach in treatment for carriers of hypertension. KEYWORDS: Hypertension; Therapeutics; Aged; Medication adherence.

\section{ADESIÓN DEL ANCIANO AL TRATAMIENTO DE LA HIPERTENSIÓN ARTERIAL SISTÉMICA: REVISIÓN INTEGRATIVA}

RESUMEN: Objetivando identificar en la literatura los factores que interfieren en el proceso de adesión al tratamiento de la hipertensión, en especial entre ancianos, fue realizada una revisión integrativa de literatura. Utilizándose las bases de datos SciELO, LILACS y PubMed, fueron elegidos 36 artículos, publicados entre enero de 2006 y diciembre de 2010, delimitándose, a partir de estes, cinco grandes grupos de factores que interfieren en la adesión al tratamiento: régimen terapéutico; aspectos socioeconómicos y demográficos; relación con los servicios y profesionales de salud; aspectos psicosociales y culturales; y apoyo familiar y social. Con base en la literatura, personas del sexo masculino y ancianos con baja renta constituyen las poblaciones más vulnerables, siendo múltiplos los aspectos que dificultan la adesión al tratamiento de la hipertensión arterial. La adopción de un abordaje multiprofesional en el tratamiento de los portadores de hipertensión se constituye en el abordaje más adecuado para promover la adesión al tratamiento. PALABRAS-CLAVE: Hipertensión; Terapéutica; Anciano; Adesión a la medicación.

\footnotetext{
*Este artigo é um recorte da pesquisa "Portadores de Hipertensão Arterial Sistêmica: representações dos pacientes a respeito da doença", fomentada pela Fundação de Amparo à Pesquisa de Minas Gerais-FAPEMIG.

${ }^{1}$ Acadêmica de Psicologia da Universidade Vale do Rio Doce. Bolsista de Iniciação Científica.

${ }^{2}$ Enfermeiro da Prefeitura Municipal de Governador Valadares. Mestre em Gestão Integrada do Território.

${ }^{3}$ Psicólogo. Doutor em Psicologia Clínica. Professor da Universidade Vale do Rio Doce.

${ }^{4}$ Cirurgiã Dentista. Doutora em Saúde Coletiva. Professora da Universidade Vale do Rio Doce.

${ }^{5}$ Economista. Doutora em Dinâmica Populacional. Professora da Universidade Federal de Minas Gerais.
}

Autor correspondente:

Leonardo Oliveira Leão e Silva

Prefeitura Municipal de Governador Valadares

Rua I, 398 - 35059-600 - Governador Valadares-MG-Brasil

E-mail: leooliveiraleao@hotmail.com
Recebido: 17/03/2011

Aprovado: 06/09/2011

Cogitare Enferm. 2012 Jan/Mar; 17(1):144-50 


\section{INTRODUÇÃO}

As doenças crônicas não-transmissíveis, dentre elas a Hipertensão Arterial Sistêmica (HAS), apresentaram um crescimento significativo nas últimas décadas, sendo responsáveis por grande número de óbitos no mundo. Dados indicam que cerca de 17 milhões dos óbitos ocorridos no ano de 2003 foram causados por complicações cardiovasculares ${ }^{(1)}$. Reconhecida como uma doença silenciosa, a HAS é a mais prevalente no mundo e, quando não tratada adequadamente, pode acarretar graves consequências e complicações, tais como infarto agudo do miocárdio e doença vascular cerebral ${ }^{(2-3)}$.

Por possuir um longo curso assintomático, sem consequências imediatas decorrentes da suspensão do tratamento, a HAS exige mudanças no estilo de vida e uso diário de medicamentos ${ }^{(4)}$. Apesar de serem conhecidas a eficácia, a efetividade e a eficiência de várias das medidas preventivas e de controle disponíveis farmacológicas ou não - provavelmente a elevação da prevalência da pressão arterial continuará, por décadas, representando um dos maiores desafios em saúde para o próprio hipertenso e para a sociedade ${ }^{(5)}$.

Dentre as dificuldades encontradas para o atendimento às pessoas hipertensas, a falta de adesão ao tratamento é reconhecida como uma das principais causas. Além desta, a adoção de práticas terapêuticas inadequadas contribui para que a maioria dos hipertensos diagnosticados não mantenha a pressão arterial sistêmica controlada ${ }^{(3)}$.

A adesão ao tratamento é considerada um processo complexo, influenciado por fatores ambientais, individuais, de acolhimento por parte dos profissionais de saúde, no qual encontram-se comprometidas as dimensões biológica, sociológica e psicológica. Tais fatores são fortes determinantes da qualidade do cuidado prestado $^{(6)}$. Além destes, somam-se os fatores socioeconômicos, culturais e comportamentais dificultando ainda mais o processo de adesão( $0^{(7)}$.

Entre os pacientes que iniciam o tratamento para o controle da HAS, 16 a 50\% abandonam a medicação anti-hipertensiva durante o primeiro ano de uso ${ }^{(8)}$. Assim, torna-se prioritário aos profissionais de saúde desenvolver e implementar estratégias que motivem o hipertenso a continuar uma determinada terapêutica, farmacológica ou não, bem como intervir junto aos pacientes como equipe multiprofissional.

Tal panorama indica que o modo como as pessoas fazem seus tratamentos (mudanças dietéticas, comportamentais, estilo de vida e, em especial, uso de medicamentos) está entre os maiores desafios no enfrentamento da HAS. O fato é que o tratamento adequado pode consistir tanto na adoção de estilo de vida saudável, quanto no uso de medicação ou a associação de ambos, com base em evidências científicas e consensos de especialistas ${ }^{(5)}$.

Considera-se que a adesão ao tratamento da HAS é fundamental para que o paciente tenha melhor qualidade de vida e menor risco de complicações cardiovasculares. Em decorrência, entende-se que manter a pressão arterial sistêmica sob controle constitui-se num indicador do cumprimento das prescrições recomendadas pelo médico e equipe de saúde. Na intenção de identificar a melhor forma de promover a adesão ao tratamento destes pacientes, delimitou-se como objetivo para o presente estudo identificar, na literatura, os fatores que interferem no processo de adesão ao tratamento da HAS, em especial, entre os idosos.

\section{MÉTODO}

Optou-se pela revisão integrativa da literatura, definida como um método por meio do qual resultados de pesquisas são sumarizados, o que possibilita síntese, categorização, análise e avaliação do conhecimento científico já produzido sobre o tema ${ }^{(9)}$. A síntese viabiliza a contextualização do pesquisador acerca da temática, além de sinalizar lacunas do conhecimento que precisam ser preenchidas com novos estudos, contribuindo para discussões a respeito dos métodos e resultados de pesquisas anteriores ${ }^{(10)}$.

Para a elaboração da revisão integrativa, no primeiro momento o revisor determina o objetivo específico, formula os questionamentos a serem respondidos e, então, realiza a busca para identificar e coletar o máximo de pesquisas primárias relevantes dentro dos critérios de inclusão e exclusão previamente estabelecidos ${ }^{(11)}$. Desta forma, para nortear a realização do presente estudo, formulou-se a seguinte questão: quais são os fatores, descritos na literatura, que interferem no processo de adesão ao tratamento da HAS, principalmente entre os idosos?

Para o levantamento bibliográfico deste estudo, realizado no último semestre de 2010, fez-se uma busca online nas bases de dados SciELO (Scientific Eletronic Library On line), LILACS (Literatura Latino-Americana e do Caribe em Ciências da Saúde) e PubMed (Public Medicine). A escolha por essas bases de dados deve-se ao fato de englobarem publicações nacionais e internacionais. 
No levantamento dos artigos, foram utilizados os seguintes descritores contextualizados ao tema em estudo: (hypertension $O R$ hipertensão $O R$ pressão alta) $A N D$ (adherence $O R$ adhesion $O R$ adesão $O R$ compliance $O R$ non-adherence $O R$ nonadherence $O R$ no-adhesion $O R$ não-adesão $O R$ observância $O R$ abandono ao tratamento). Salienta-se que foram utilizados os descritores 'observância' e 'abandono ao tratamento' como sinônimos para adesão e não-adesão, respectivamente.

De acordo com os critérios de inclusão, os artigos deveriam estar disponíveis nas bases de dados indicadas e estritamente voltados ao objeto de estudo. Publicados entre janeiro de 2006 e dezembro de 2010, nos idiomas português, inglês e espanhol, apresentando resumos e textos na íntegra. Para verificar se os artigos atendiam aos critérios de inclusão, realizou-se uma avaliação por dois revisores independentes, sendo as avaliações subsequentemente comparadas.

Após leitura dos artigos selecionados, as informações foram coletadas e digitadas em banco de dados de acordo com as seguintes variáveis: ano, características metodológicas do estudo, autores, objetivos do trabalho, sujeitos estudados - quanto à idade e sexo, resultados encontrados e conclusão/considerações finais do estudo. Todos os registros foram armazenados em um arquivo específico do programa Microsoft ${ }^{\circledR}$ Excel 2007.

\section{RESULTADOS}

Foram identificadas no SciELO 652 referências. Após uma primeira análise baseada nos critérios de inclusão, leitura dos resumos disponíveis e avaliação sobre o questionamento elaborado, foram selecionados 12 artigos. No LILACS foram identificadas 180 referências e, seguindo-se os mesmos critérios, foram considerados 18 artigos. No PubMed foram identificadas 2121 referências, sendo considerados 22 artigos. Ressalta-se que 16 foram encontrados em ambas as bases de dados, sendo estes considerados apenas uma vez no cômputo dos textos incluídos no estudo. Desta forma, 36 artigos fizeram parte da presente revisão.

Quanto ao delineamento do estudo, 30 (83\%) eram do tipo transversal, 6(17\%) do tipo prospectivo. Dentre eles, $18(50 \%)$ estavam estruturados na língua portuguesa, 11(31\%) na inglesa e 7(19\%) na espanhola. Em relação ao ano de publicação, $7(19 \%)$ foram publicados em 2010; 11(31\%) em 2009; 5(14\%) em 2008; 10(28\%) em 2007 e 3 (8\%) em 2006. A maioria foi publicada no
Brasil, 20 (55\%) e os demais em Cuba, 3(8\%); EUA, 2(6\%); Turquia, 2(6\%); e outros países, $9(25 \%)$. Estes dados estão sintetizados na tabela 1 .

A partir dos textos estudados, foram identificados cinco grupos de fatores que interferem no processo de adesão ao tratamento entre pacientes idosos: Regime terapêutico, 13(36\%) artigos integram este grupo; Aspectos socioeconômicos e demográficos, 7(19\%); Relação com os serviços e profissionais de saúde, 7(19\%); Aspectos psicossociais e culturais, 7(19\%); Apoio social e familiar, 2(6\%).

1. Regime terapêutico: observou-se que a grande quantidade de medicamentos, variadas doses e inadequado conhecimento acerca da terapêutica dificultam a adesão ao tratamento. Os estudos deste grupo apontam a educação em saúde como excelente ferramenta no processo de redução do impacto destas variáveis no nível de adesão.

2. Aspectos socioeconômicos e demográficos: identificados como altamente relevantes. Indivíduos do sexo masculino, idade avançada, baixa condição socioeconômica e de escolaridade estão associados a menor adesão ao tratamento. Por outro lado, estudos indicam a idade mais avançada como um facilitador do processo de adesão. No entanto, esses não consideram as variáveis fisiológicas (como exemplo o esquecimento) que podem interferir no processo de adesão.

\section{Relação com os serviços e profissionais de saúde:} identificada nos estudos como grande promotora da adesão ao tratamento. Deste grupo fazem parte a qualidade do serviço de saúde, a inserção das unidades com Estratégia Saúde da Família (ESF) e uma relação agradável e de escuta constante dos profissionais com os usuários.

4. Aspectos psicossociais e culturais: os pacientes hipertensos possuem maiores dificuldades de aderir às mudanças comportamentais necessárias ao tratamento. Além disso, a experiência anterior com uma pessoa de seu convívio social com a doença não a estimula a adotar as condutas preconizadas. Por outro lado, suas crenças e conhecimentos culturais em relação às medidas de prevenção e remédios populares melhoram a adesão à terapêutica. No entanto, tal conhecimento adquirido no convívio social não garante um eficaz controle da pressão arterial.

5. Apoio social e familiar: os estudos indicam que o familiar ou cuidador devidamente capacitado e com conhecimento adequado sobre a HAS constitui-se num grande facilitador do processo de adesão ao tratamento. 
Tabela 1 - Identificação dos estudos selecionados para revisão integrativa sobre fatores que interferem na adesão ao tratamento de hipertensão, 2006-2011. Governador Valadares, 2011

\begin{tabular}{|c|c|c|c|}
\hline AUTOR & ANO DE PUBLICAÇÃO & PAÍS & METODOLOGIA \\
\hline Lima et al. & 2010 & Brasil & Estudo descritivo, transversal, exploratório \\
\hline Helena et al. & 2010 & Brasil & Estudo descritivo, transversal \\
\hline Oshiro et al. & 2010 & Brasil & Estudo de coorte, transversal \\
\hline Duarte et al. & 2010 & Brasil & Estudo de coorte, prospectivo \\
\hline Santos et al. & 2010 & Brasil & Estudo transversal \\
\hline Morgado et al. & 2010 & Portugal & Estudo transversal \\
\hline Holt et al. & 2010 & EUA & Estudo de coorte, prospectivo \\
\hline Moreira et al. & 2009 & Brasil & Estudo descritivo, transversal \\
\hline Milián et al. & 2009 & Cuba & Estudo transversal \\
\hline Baldissera et al. & 2009 & Brasil & Estudo descritivo, transversal, observacional \\
\hline Veras et al. & 2009 & Brasil & Estudo descritivo, transversal \\
\hline Estadístico et al. & 2009 & Colômbia & Estudo de coorte, descritivo, transversal \\
\hline Dosse et al. & 2009 & Brasil & Estudo descritivo, transversal \\
\hline Bloch et al. & 2009 & Brasil & Estudo descritivo, observacional, transversal \\
\hline Reiners et al. & 2009 & Brasil & Estudo de coorte, descritivo, transversal \\
\hline Karaeren et al. & 2009 & Turquia & Estudo transversal \\
\hline Uzun et al. & 2009 & Turquia & Estudo descritivo, transversal \\
\hline Shah et al. & 2009 & EUA & Estudo de coorte retrospectivo \\
\hline Melano-Carranza et al. & 2008 & México & Estudo transversal \\
\hline Pires et al. & 2008 & Brasil & Estudo descritivo, transversal \\
\hline Xavier et al. & 2008 & Brasil & Estudo de coorte histórico, transversal \\
\hline Paternina et al. & 2008 & Cuba & Estudo descritivo, transversal \\
\hline Mizuno et al. & 2008 & Japão & Estudo de coorte, prospectivo \\
\hline Sales et al. & 2007 & Brasil & Estudo observacional, transversal \\
\hline Mochel et al. & 2007 & Brasil & Estudo analítico, transversal \\
\hline Araújo et al. & 2007 & Brasil & Estudo observacional, prospectivo \\
\hline Taveira et al. & 2007 & Brasil & Estudo descritivo, exploratório, transversal \\
\hline Saraiva et al. & 2007 & Brasil & Estudo descritivo, exploratório, transversal \\
\hline Matos et al. & 2007 & Cuba & Estudo descritivo, transversal \\
\hline Saraiva et al. & 2007 & Brasil & Estudo descritivo, exploratório, transversal \\
\hline Neutel et al. & 2007 & Canadá & Estudo de coorte, prospectivo \\
\hline Dean et al. & 2007 & Inglaterra & Estudo transversal \\
\hline Hashmi et al. & 2007 & Paquistão & Estudo transversal \\
\hline Tuesca-Molina et al. & 2006 & Espanha & Estudo transversal \\
\hline Mendonza et al. & 2006 & Chile & Estudo observacional, transversal \\
\hline Faé Et Al. & 2006 & Brasil & Estudo descritivo, exploratório, transversal \\
\hline
\end{tabular}




\section{DISCUSSÃO}

Um estudo realizado com 353 hipertensos indicou que os principais motivos que contribuem para que o paciente abandone o tratamento são: o alto custo dos medicamentos, a necessidade de tomá-lo várias vezes ao dia, a ocorrência de efeitos indesejáveis, o desconhecimento das complicações, a ausência de sintomatologia e o esquecimento ${ }^{(12)}$. Soma-se a esses fatores o fato de que, quando o processo de conscientização é negligenciado pode em incorrer uso incorreto do medicamento e levar o paciente a não seguir as prescrições médicas de maneira satisfatória ${ }^{(13-14)}$.

Em decorrência, como propostas para melhorar o regime terapêutico e aumentar a adesão ao tratamento, autores indicam melhor avaliação inicial para evitar constantes mudanças de esquema terapêutico e, sempre que possível, a prescrição de menor número de medicamentos ${ }^{(14)}$. Por último, é relatada a necessidade de educação e informações claras ao paciente acerca da doença e possíveis complicações, bem como acompanhamento periódico desse por meio de consultas.

Em trabalho realizado com 72 hipertensos cadastrados e acompanhados no Centro de Saúde Escola concluiu-se que a maioria dos entrevistados não aderiu ao tratamento não-farmacológico, principalmente por encontrarem dificuldades no cumprimento da dieta e da prática de atividade física. Este fato pode estar associado ao entendimento de que as restrições da dieta, por exemplo, a diminuição da ingestão de sódio, denotam a ideia de 'castigo' por estarem ligadas ao prazer em degustar alimentos, ou ainda, associado à perda da liberdade de escolha ${ }^{(15)}$.

Muitos pesquisadores relacionam a adesão ao tratamento com adesão à medicação ${ }^{(16)}$. Porém, é importante destacar a imprescindível necessidade de adotar outros comportamentos inerentes à saúde que vão além do seguimento da prescrição de medicamentos. Deve-se pensar em um tratamento que considere os aspectos referentes ao sistema de saúde, aos fatores socioeconômicos, e qualidade de vida do idoso ${ }^{(17)}$. Também nas dificuldades próprias de se cumprir qualquer forma de tratamento, as características e cultura dos pacientes, e a forma de manifestação da própria doença.

Nesta linha de raciocínio, estudos indicam as condições socioeconômicas como preditoras da não adesão ao tratamento ${ }^{(18)}$, quanto mais baixas, menores são as taxas de adesão ao tratamento anti-hipertensivo, pois é menor o conhecimento da doença e mais difícil o acesso aos serviços de saúde.
Algumas das pesquisas indicam que a relação entre o profissional de saúde e o paciente constitui-se em fator influenciador da adesão ao tratamento ${ }^{(7,19)}$. Segundo os estudos, alguns profissionais abrem mão da estratégia de conscientização e de seu papel de educador e de auxiliar no tratamento. Dessa maneira, o acompanhamento ao paciente tem sido realizado de maneira inadequada. Por força deste abandono, o usuário realiza o tratamento a seu modo, o que pode ser prejudicial à sua saúde.

Outro ponto a se destacar é a crença em saúde, por ser esta capaz de influenciar a adesão ao tratamento da $\mathrm{HAS}^{(6)}$. Num estudo realizado com 263 hipertensos, no qual buscou-se identificar os fatores psicossociais relacionados com a adesão ao tratamento, concluiu-se que as crenças do paciente, o conhecimento adequado sobre as características da doença, a eficácia do tratamento e a percepção de redes adequadas de apoio social são comumente facilitadoras da adesão ${ }^{(20)}$.

A crença no tratamento é, por si só, um forte preditor do comportamento para busca do seguimento adequado $^{(6)}$. Assim, muito embora a percepção dos benefícios quanto à adoção de medidas de prevenção e controle da HAS não implicarem, necessariamente, a coerência e efetividade de ações em busca da adesão. É muito importante fornecer ao paciente elementos para que possa compreender o tratamento levando-o a acreditar que terá resultados positivos se seguí-lo de forma adequada.

Os fatores psicossociais, como a experiência de vida, são apontados como determinantes para adesão ao tratamento $^{(21)}$. Contudo, em oposição a esta asserção, em um estudo realizado com 18 hipertensos da área de abrangência da ESF, constatou-se que a adesão às medidas de controle eram muito baixas, até mesmo entre aqueles que sofreram complicações decorrentes da $\mathrm{HAS}^{(22)}$. Isto indica que a experiência de uma complicação decorrente da hipertensão não assegura maior adesão do paciente ao tratamento. Os resultados encontrados demonstram as dificuldades que os pacientes hipertensos têm em adotar medidas de controle e a necessidade de desenvolvimento de trabalhos de educação em saúde que permitam a transformação dessa realidade.

Dentre os fatores identificados que interferem no tratamento, os mais favoráveis à adesão dos idosos avaliados foram a motivação pelo prolongamento da vida e a ajuda do familiar consciente no cumprimento da terapêutica prescrita. Essa realidade demonstra o grande potencial do familiar do idoso nas estratégias de promoção da saúde ao se engajar no tratamento, 
passa a conhecer as medidas de prevenção e cuidado da hipertensão. Tal conhecimento poderia também ser utilizado pelo familiar no seu próprio cuidado.

O familiar que assume o papel de cuidador do paciente hipertenso, assim como o hipertenso, não têm conhecimento adequado ou suficiente da doença ${ }^{(23)}$. Neste sentido, outros estudos evidenciam que o pouco conhecimento dos familiares sobre a doença faz com que não se envolvam no processo de tratamento, sobretudo quando não existe dependência do idoso ${ }^{(23-24)}$.

Nesta perspectiva, existem evidências de que a participação do familiar não é o fator determinante para a adesão ao tratamento, mas, quanto maior o vínculo afetivo envolvido na relação, maior será sua participação na provisão do cuidado. Diante desses resultados, os estudos indicam a educação em saúde como instrumento fortalecedor do regime terapêutico ${ }^{(25)}$ dado que a adoção desta medida promove maior interação entre familiares e profissionais de saúde, contribuindo para que o tratamento se torne mais efetivo e seguro.

Em meio aos fatores indicados aqui como intervenientes no processo de adesão ao tratamento da HAS por idosos pode-se afirmar que a ocorrência da adesão implica em mudanças nos comportamentos relacionados aos cuidados para com a saúde. O hipertenso precisa primeiramente valorizar os benefícios das mudanças no seu estilo de vida. Para isto, deve acreditar que é possível reduzir a severidade da doença, e isso só será possível a partir do momento em que reconhecer que precisa alterar alguns de seus comportamentos.

Atenção especial deve ser dada aos resultados alcançados com as pesquisas que tratam do assunto, sobretudo em função do processo de transição demográfica enfrentado pelo Brasil; a população está se tornando mais idosa ${ }^{(26)}$. Essa transição traz consigo a transição epidemiológica em que as doenças crônicas não transmissíveis tornam-se mais prevalentes. Tal perspectiva indica a necessidade da efetivação de políticas públicas em saúde que valorizem o idoso na promoção de ações que contribuam para maior adesão ao tratamento da hipertensão.

\section{CONSIDERAÇÕES FINAIS}

A realização deste estudo possibilitou identificar que a adesão ao tratamento é um processo complexo que envolve fatores ambientais, emocionais e sociais. Em outros termos, o conhecimento correto do paciente ou de seu cuidador sobre a HAS; a condição socioeconômica (1 a 3 salários mínimos) e demográficas (sexo masculino); crenças e expectativas sobre a melhoria de suas condições com o tratamento; empatia do paciente para com o profissional e compromisso do profissional de saúde com o paciente; apoio social e envolvimento da família no tratamento formam um rol dos principais fatores que interferem no processo de adesão ao tratamento da HAS.

Aidna, os estudos revelam que há maior facilidade por parte dos pacientes idosos em aderir ao tratamento medicamentoso do que cumprir prescrições que exijam mudanças comportamentais e de estilo de vida.

Por fim, sendo o idoso mais propenso à HAS, deve-se dar especial atenção aos fatores que, segundo a literatura, interferem no processo de adesão destes ao tratamento. Isto é, objetivando promover melhor qualidade de vida para este grupo populacional, os profissionais de saúde, inseridos em uma equipe multiprofissional, devem atentar para o relacionamento dos familiares e cuidadores com o hipertenso; o nível de conhecimento destes sobre a HAS; a quantidade de medicações ingeridas; o esquecimento; e as mudanças de estilo de vida, tais como o seguimento da dieta e prática de atividades físicas.

\section{REFERÊNCIAS}

1. Organização Pan-Americana da Saúde (OPAS). Doenças crônico-degenerativas e obesidade: estratégia mundial sobre alimentação saudável, atividade física e saúde. Brasília: OPAS; 2003.

2. Lessa I. Hipertensão arterial sistêmica no Brasil: tendência temporal. Cad. saude publica. 2010;26(8):470-71.

3. Peres DS, Magna JM, Viana LA. Arterial hypertension patients: attitudes, beliefs, perceptions, thoughts and practices. Rev. saude publica. 2003;37(5):635-42.

4. Miranda RD, Perrotti TC, Bellinazzi VR, Nóbrega TM, Cendoroglo MS, Neto JT. Hipertensão arterial no idoso: peculiaridades na fisiopatologia, no diagnóstico e no tratamento. Rev. bras. hipertens. 2002;9(3):293-300.

5. Lessa Í, Magalhães L, Araujo MJ, Filho NA, Aquino E, Oliveira MMC. Hipertensão arterial na população adulta de Salvador (BA) - Brasil. Arq. bras. cardiol. 2006;87(6):747-56.

6. Pires CGS, Mussi FC. Crenças em saúde para o controle da hipertensão arterial. Cienc. saude colet. 2008;13(2):2257-67.

7. Helena ETS, Nemes MI, Eluf-Neto J. Avaliação da assistência a pessoas com hipertensão arterial em 
unidades de estratégia saúde da família. Saude soc. 2009;19(3):614-26.

8. Riera ARP. Hipertensão arterial: conceitos práticos e terapêutica. São Paulo: Atheneu; 2000.

9. Ursi ES. Prevenção de lesões de pele no perioperatório: revisão integrativa da literatura [dissertação]. Ribeirão Preto (SP): Universidade de São Paulo; 2005.

10. Polit DF, Beck CT. Using research in evidence-based nursing practice. In: Polit DF, Beck CT, editors. Essentials of nursing research. Methods, appraisal and utilization. Philadelphia: Lippincott Williams \& Wilkins; 2006. p. 457-94.

11. Beyea SC, Nicoll LH. Writing an integrative review. AORN j. 1998;67(4):877-80.

12. Mion JRD, Pierin AMG. Causas de baixa adesão ao tratamento e o perfil de pacientes hipertensos. In: Congresso da Sociedade Brasileira de Hipertensão; 1996. p. 120; São Paulo: Sociedade Brasileira de Hipertensão; 1996.

13. Gusmão JL, Ginani GF, Silva GV, Ortega KC, Júnior DM. Adesão ao tratamento em hipertensão arterial sistólica isolada. Rev. bras. hipertens. 2009;16(1):38-43.

14. Fae AB, Oliveira ERA, Silva LT, Cadê NV, Mezadri VA. Facilitadores e dificultadores da adesão ao tratamento da hipertensão arterial. Rev. enferm. 2006;14(1):32-6.

15. Baldissera VDA, Carvalho MDB, Pelloso SM. Adesão ao tratamento não-farmacológico entre hipertensos de um centro de saúde escola. Rev. gaúcha enferm. 2009;30(1):27-32.

16. Haynes RB. Determinants of compliance: The disease and the mechanics of treatment. Baltimore MD, Johns Hopkins University Press; 1979.

17. Holt EW, Muntner P, Joyce CJ, Webber L, Krousel-Wood MA. Health-related quality of life and antihypertensive medication adherence among older adults. Age Ageing. 2010; 39(4):481-7.

18. Machado CA. Adesão ao tratamento - tema cada vez mais atual. Rev. bras. hipertens. 2008;15(4):220-21.

19. Reiners AAO, Nogueira MS. Conscientização do usuário hipertenso para a adesão ao tratamento. Rev latino-am. enfermagem. 2009;17(1):59-65.

20. Matos RY, Alfonso LM, Bayarre VH. Adherencia terapéutica y factores psicosociales en pacientes hipertensos. Rev Cubana Med Gen Integr [Internet] 2007; 23(1) [acesso em 12 mai 2011]. Disponível em: http://scielo.sld.cu/scielo.php?script=sci_arttext\&pid $=$ S0864-21252007000100005\&lng=es.

21. Araújo JC, Guimarães AC. Controle da hipertensão arterial em uma unidade de saúde da Família. Rev. saude publica. 2007;41(3):368-74.

22. Sales CM, Tamaki ED. Adesão às medidas de controle da hipertensão arterial sistêmica: o comportamento do hipertenso. Cogitare enferm. 2007;12(2):157-63.

23. Saraiva KRO, Santos ZMSA, Landim FLP, Lima HP, Sena VL. O processo de viver do familiar cuidador na adesão do usuário hipertenso ao tratamento. Texto contexto enferm. 2007;16(1):63-70.

24. Contiero AP, Pozati MPS, Challouts RI, Carreira L, Marcon SS. Idoso com hipertensão arterial: dificuldades de acompanhamento na estratégia saúde da família. Rev. gaúcha enferm. 2009;30(1):62-70.

25. Júnior DPL, Amaral RT, Veiga EV, Cárnio EC, Nogueira MS, Pelá IR. Farmacoterapia no idoso: revisão sobre a abordagem multiprofissional no controle da hipertensão arterial sistêmica. Rev. latino-am. enfermagem. 2006;14(3):435-41.

26. Carvalho JAM, Rodriguez-Wong LL. A transição da estrutura etária da população brasileira na primeira metade do século XXI. Cad. saude publica. 2008;24(3):597-605.

Cogitare Enferm. 2012 Jan/Mar; 17(1):144-50 